\title{
Performance comparison of complementary and AND subtraction detection techniques for hybrid SCM SAC-OCDMA system
}

\begin{abstract}
The subtraction detection techniques for a hybrid SCM spectral-amplitude-coding (SAC) OCDMA using double weight (DW) code family are investigated. The experimental simulation results show that the proposed new subtraction detection technique provides a better system performance.

Keyword: Subtraction detection techniques; Double weight (DW) code family; Hybrid SCM SAC-OCDMA system
\end{abstract}

